\title{
Encephalopathy in Liver Transplantation: Neuropathology and CMV Infection
}

\author{
C. Power, S.D. Poland, K.H. Kassim, J.C.E. Kaufmann and G.P.A. Rice
}

\begin{abstract}
The clinical histories and pathological findings of 27 autopsied cases of orthotopic liver transplantation (OLT) were reviewed. Fatal OLT was complicated in $93 \%$ of cases by neurological dysfunction, usually manifested by encephalopathy, with or without seizures. The etiology of the encephalopathy was largely multifactorial (44\%) or undetermined (20\%). Subarachnoid hemorrhage, central pontine myelinolysis, meningitis, brain infarction, polyclonal B cell lymphoma and spinal cord necrosis were common neuropathological findings. These diagnoses were often masked by other systemic illnesses. The role of cytomegalovirus (CMV) in neurologic dysfunction was explored with in situ hybridization and immunohistochemical techniques. OLT cases showed a significantly higher (89\%) frequency of CMV genomic material in brain tissue compared to age-matched non immunocompromised (NIC) patients (23\%). All OLT cases with encephalopathy of undetermined cause demonstrated unusually prominent hybridization to the CMV probe. CMV may be an important cause of encephalopathy in such patients.
\end{abstract}

RÉSUMÉ: Encéphalopathie dans la transplantation hépatique: neuropathologie et infection à CMV Nous avons revisé l'histoire clinique et les constations anatomopathologiques de 27 cas de transplantation hépatique orthotopique (THO) ayant subi une autopsie. Une THO fatale était compliquée dnns $93 \%$ des cas par une dysfonction neurologique se manifestant habituellement par une encéphalopathie avec ou sans convulsion. L'étiologie de l'encéphalopathie était la plupart du temps d'origine multifactorielle $(44 \%)$ ou indéterminée $(20 \%)$. Les constations neuropathologiques les plus fréquentes étaient l'hémorragie sous-arachnoïdienne, la myélinolyse, la méningite, l'infarctus cérébral, le lymphome polyclonal à cellule B et la nécrose de la moelle épinière. Ces diagnostics étaient souvent masqués par d'autres maladies systémiques. Le rôle du cytomégalovirus (CMV) dans la dysfonction neurlogique a été investiqué par l'hybridation in situ et par des techniques immunohistochimiques. Les cas de THO présentaient une fréquence significativement plus élevée (89\%) de matériel génomique CMV dans le tissu cérébral comparés à des patients non-immunocompromis appariés poue l'âge (23\%). Tous les cas de THO avec encéphalopathie de cause indéterminée présentaient un degré particulièrement marqué d'hybridation avec le sonde CMV. Le CMV peut être une cause importante d'encéphalopathie chez ces patients.

Can. J. Neurol. Sci. 1990; 17:378-381

Orthotopic liver transplantation (OLT) is the optimal treatment for terminal liver failure. ${ }^{1}$ Neurological complications including encephalopathy and seizures arise in liver transplant patients. ${ }^{2,3}$ Little is known about the etiological and pathological features of these complications. Because OLT may be associated with multisystem failure and immunosuppression, the diagnosis of neurological complications is difficult. To determine the neurological complications encountered following OLT, and their neuropathological features, we embarked upon a retrospective study of 27 fatal OLT cases.

CMV infection is a common systemic complication of OLT. ${ }^{4}$ CMV encephalitis has been described in immunosuppressed patients. 5,6,7 We examined the role of CMV as a cause of neurological dysfunction in these patients with an in situ hybridization technique.

\section{Materials and Methods}

Between July 1977 and September 1988, 130 OLT were performed. Of the 32 OLT cases who died, $27(84 \%)$ underwent autopsy with review of the clinical records. Brains and spinal cords were studied after fixation for one week in $20 \%$ formalin. Standard sections were taken from frontal, temporal and occipital cortices, basal ganglia, thalamus, hippocampus, cerebellum, brain stem and spinal cord. All paraffin-embedded sections were stained with hematoxylin and eosin and with special stains (Solochrome R/Cresyl Violet, Bielchowsky, Masson's Trichrome).

\section{In Situ Hybridization}

Using a biotinylated CMV DNA probe (Enzo Biochem, New York) in situ hybridization was performed on sections from cortex and brain stem following deparaffinization (Hemo De, Fisher, Don Mills). Sections were rehydrated through graded ethanol washes, immersed in $3 \% \mathrm{H}_{2} \mathrm{O}_{2}$ in $100 \%$ methanol for 15 minutes to block endogenous peroxidase activity, rinsed for 5 minutes in water at $20^{\circ} \mathrm{C}$, covered with proteinase $\mathrm{K}(0.1 \mathrm{mg} / \mathrm{ml})$ and incubated for 10-15 minutes at $37^{\circ}$.

The biotinylated CMV specific DNA probe was added to the section, incubated at $95^{\circ}$ for $3-5$ minutes and then at $20^{\circ}$ for

From the Department of Clinical Neurological Sciences, University of Western Ontario, London

Received May 23, 1990. Accepted in final form August 16, 1990

Reprint requests to: Dr. G. Rice, University Hospital, 339 Windermere Road, London, Ontario, Canada N6A 5A5 
30 minutes. The slides were then washed and covered with an avidin-biotin horseradish peroxidase for 20 minutes, rinsed in wash buffer and covered with $2 \% 3$ amino-9-ethylcarbazole, $3 \%$ $\mathrm{H}_{2} \mathrm{O}_{2}$ in $0.05 \%$ sodium acetate buffer for 10 minutes and counter stained with $0.2 \%$ fast green.

The controls for CMV infection included tissues known to be infected with CMV (adrenal gland), and a human fetal foreskin fibroblasts infected in vitro with CMV. Uninfected fibroblasts were also used as controls. To study the frequency of CMV infection in nonimmunocompromised (NIC) patients, brain tissue (cortex and brainstem) from 30 age-matched patients who had died from miscellaneous acute neurological causes (trauma, hemorrhage and ischemia) was probed for CMV.

The CMV probe consisted of two clones of CMV DNA cloned into the Bam HI site of pBR322, representing about $17 \%$ of the total CMV genome. It has been shown to specifically label CMV infected cells in paraffin-embedded tissues. ${ }^{8}$

A biotinylated hepatitis B virus specific DNA probe (Enzo Biochem, New York) was also tested concomitantly with the CMV probe on controls and positive OLT patients. The same hybridization technique was used.

Immunoperoxidase staining for CMV specific antigen was performed on CMV infected controls and OLT cases using a technique described previously. ${ }^{9}$

All slides were interpreted by three microscopists without knowledge of the patient identity. The number of positive cells per slide was averaged from all slides of the same case. A statistical analysis (Chi-square) was performed to compare the frequency of CMV in OLT patients versus the 30 age matched controls.

\section{RESULTS}

\section{Clinical Features}

There were 17 females and 10 males with a mean age of 37.7 years (12-64 years). The major original liver diseases included primary biliary cirrhosis $(n=5)$, chronic active hepatitis $(n=5)$, Budd-Chiari syndrome $(n=4)$, primary sclerosing cholangitis $(n=3)$, non $A$, non $B$ hepatitis $(n=2)$ and alpha-1antitrypsin deficiency $(n=2)$. The mean survival time after OLT was 48 days (5-270 days). Five patients underwent repeat OLT. All patients received immunosuppressive medications (corticosteroids, cyclosporin A, antilymphocytic globulin, azathioprine and OKT3). Systemic complications were frequent with sepsis being the most common. The causes of sepsis (positive blood, sputum or urine culture) included bacterial $(n=19)$, fungal $(n=7)$ and viral $(n=10)$. Nine of the viral infections were due to CMV. Other major systemic complications included coagulopathy $(n=19)$, thrombocytopenia $(n=17)$, renal failure $(n=13)$ liver failure (rejection, infection or drug induced; $n=18)$ and hypomagnesemia $(n=20)$.

Preterminal CNS complications arose in 25 patients and were the presumed cause of death in 4 patients (Table 1). The deaths, directly related to CNS disease, were due to meningitis $(n=2)$, hemorrhage $(n=1)$ and lymphoma metastatic to the CNS $(n=1)$.

A preterminal encephalopathy developed in 25 patients. Based on clinical records, encephalopathy was stratified into 3 categories: mild encephalopathy (drowsiness) $(n=4)$, moderate (stupor, disorientation, psychosis) $(\mathrm{n}=5)$ and severe (coma) $(n=16)$. Etiologies for the encephalopathy are outlined in Table 1.
Table 1. Preterminal Neurological Complications

Number of Cases

Encephalopathy (causes)
Multifactorial (hypoxia, hepatic or renal
failure, sepsis)
Intracranial hemorrhage
Sepsis
Cyclosporin toxicity
CNS lymphoma
Meningitis
Undetermined
TOTAL

TOTAL

Seizures (all patients were also encephalopathic)

Generalized Tonic-clonic

Partial motor

TOTAL

$8(30 \%)$

In five cases with encephalopathy (mild $n=1$, moderate $n=3$ and severe $n=1$ ), no major cause was identified.

Seizures occurred in 8 patients: 6 were generalized (tonicclonic) and 2 were focal. All patients with seizures were hypomagnesemic (mean: $0.58 \mathrm{mmol} / \mathrm{l}$ ) at the time of their seizures. In all patients with seizures, other metabolic and/or structural abnormalities were identified by neuropathological study (hemorrhage, infarction and infection).

Meningitis occurred in 5 patients although only two cases were diagnosed antemortem (Klebsiella pneumoniae, Cryptococcus neoformans). The three post-mortem cases included meningitides due to E. coli, H. zoster and an undiagnosed infection.

Intracranial hemorrhage occurred in 6 patients although this diagnosis was only made in 3 patients before death. The 3 antemortem recognized hemorrhages occurred in the thalamus, internal capsule and subarachnoid space; the remaining hemorrhages diagnosed at autopsy were all subarachnoid. Three of six patients with hemorrhage were encephalopathic due to multiple causes and had either thrombocytopenia or a coagulopathy.

\section{Pathology}

Vascular changes were the most common neuropathologicall findings. Aside from the 6 gross hemorrhages, petechial hemorrhages were noted in 7 other patients (though not recognized clinically) in the cortex $(n=6)$ and brain stem $(n=1)$. Infarction was seen in 6 patients ( 1 cortex, 1 midbrain and 4 pituitary) again without any clinical evidence to suggest such lesions. Ischemic-anoxic changes were observed in 20 patients.

Metabolic abnormalities such as Alzheimer Type II astrocytes were common $(n=18)$. Central pontine myelinolysis $(C P M)$ was observed in 5 patients. All cases were hyponatremic during their postoperative period but CPM was not diagnosed antemortem.

Along with the 5 meningitides, 2 abscesses (Cryptococcus neoformans, Klebsiella pneumoniae) were found. Microglial nodules (MGN) were observed in 7 patients. They were found in cortical and subcortical tissue. Viral inclusions were not seen.

Metastatic polyclonal B cell lymphoma was seen in the CNS of two patients. Both patients demonstrated kappa and lambda light chain immunoreactivity. One patient was suspected to have systemic and CNS B cell lymphoma though not confirmed antemortem; the second patient's lymphoma was diagnosed post mortem. Spinal cord necrosis was found in two cases. 


\section{In Situ Hybridization}

The results of the in situ hybridization are shown in Figure 1. Twenty-four OLT $(89 \%)$ and 7 NIC (23\%) patients were positive for CMV. The CMV probe hybridized in the cortex, subcortical white matter and brain stem. CMV was usually scattered in the deep parenchyma and clustered in the perivascular regions. Staining was concentrated in the nuclei of astrocytes, neurons, oligodendrocytes, ependymal, endothelial and perivascular cells (Figure 2). The regional distribution of CMV infected cells was studied in 10 OLT patients found to have marked CMV hybridization. Positive CMV cells were most frequently found in the cerebellar and cerebral cortices and least frequently in the spinal cord (Table 2). OLT patients with MGN averaged greater than 10 cells per slide by in situ hybridization. The frequency of CMV staining in OLT cases was significantly different for that in the NIC patients (Chi square $=27.3, \mathrm{p}<.005)$.

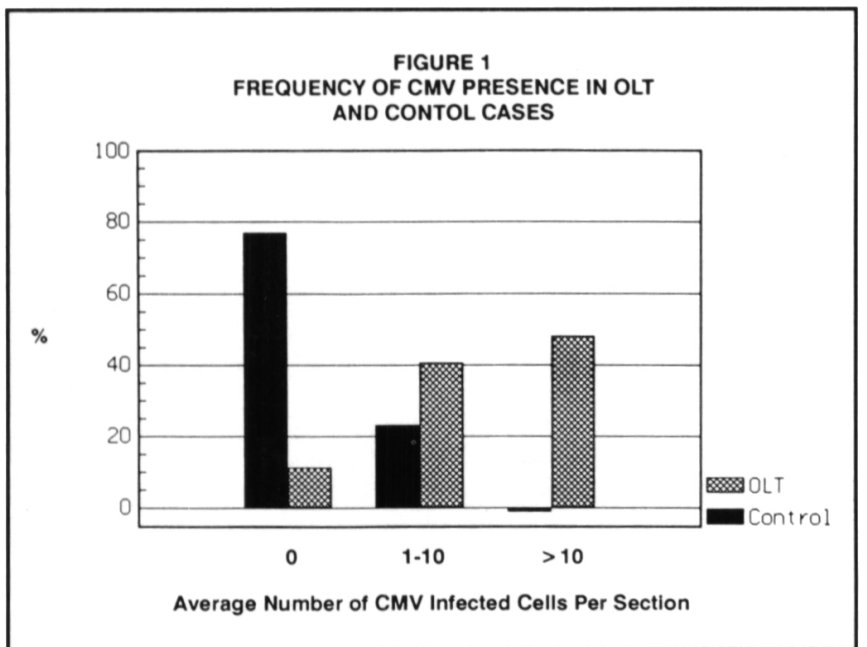

Figure I - The percentage of OLT and control cases showing hybridization with CMV probe.

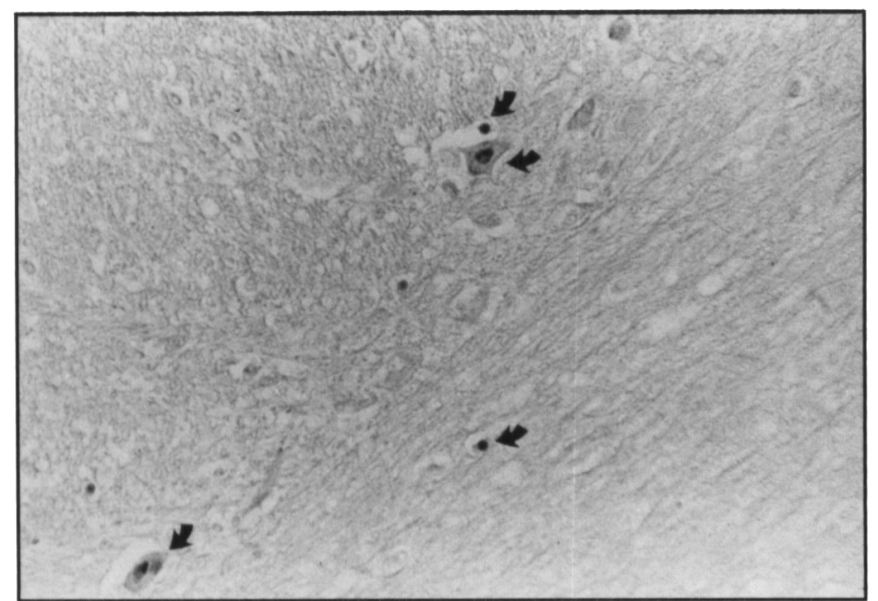

Figure 2 - Neurons and oligodendrocytes showing CMV probe hybridization. (Dark nuclei with arrow's representing CMV positive cells) $\times 200$.

In the five patients whose encephalopathy was clinically undetermined, in situ hybridization revealed more than $10 \mathrm{CMV}$-positive cells per slide.

Table 2. CMV Localization (10 patients)

Site

Average Number Cells/Slide/Patient

cerebellum (cortex)

cerebrum (cortex and adjacent white matter)

brain stem

basal ganglia

hippocampus

spinal cord

10

9

8.5

7

6.5

4.5

The hepatitis B virus specific DNA probe did not bind in the CMV infected control and OLT slides. The uninfected control slides did not show CMV or HBV probe binding. The immunoperoxidase staining for CMV showed positive cells on the control slides but on none of the 10 OLT cases tested (all were positive for CMV by in situ hybridization).

\section{Discussion}

Over $90 \%$ of cases of fatal OLT are complicated by a preterminal encephalopathy. The clinical features were variable. The etiology was generally multifactorial $(44 \%)$ : hepatic failure, anoxia, sepsis. However, in $20 \%$ of cases, central nervous system (CNS) dysfunction appeared to be secondary to a primary CNS event such as meningitis, B cell lymphoma or intracranial hemorrhage.

Seizures often accompanied the encephalopathy. In all cases in which hypomagnesemia or cyclosporine toxicity were suspected to be the primary cause, other structural or inflammatory lesions were identified at post mortem. We conclude that seizures in OLT patients may often occur due to focal brain injury but metabolic abnormalities may predispose these patients to seizures as suggested in earlier reports. ${ }^{10}$

The higher frequency of encephalopathy observed in our cases as compared to other studies, 2,3 reflects the severity of the patients' general medical condition. Patients with unexplained encephalopathy have not been reported in earlier studies.

An unusually high percentage of unsuspected diagnoses was found at autopsy. CPM, infarction, hemorrhage, meningitis, spinal cord necrosis and CNS lymphoma were not suspected by the attending physicians. The diagnosis was likely confounded by the encephalopathy. In previous studies, the frequency of clinically unrecognized conditions disclosed at autopsy was not discussed.

We were intrigued by the significant frequency of CMV hybridization in the CNS of OLT patients in contrast to the NIC patients. CMV presence in the CNS of large numbers of transplant patients has not been previously demonstrated. Occasional cases of CNS CMV infection in transplant patients have been reported without molecular biological or immunological supporting evidence. ${ }^{5,6,7}$ However CMV is a well recognized opportunistic CNS infection in other immunocompromised patients especially in those with acquired immunodeficiency syndrome (AIDS). ${ }^{11,12}$

CMV hybridization in our study was often colocalized with microglial nodules (MGN). MGN's represent the hallmark of active $\mathrm{CMV}$ infection in patients with encephalopathy ${ }^{13}$ and are frequently colocalized with CMV positive cells. ${ }^{14}$ This suggests that CMV in OLT patients has an active infective role. This is 
also supported by our finding that all 9 OLT cases with active systemic CMV infection showed CMV probe hybridization in brain tissue. The OLT patients with unexplained encephalopathy, demonstrated marked CMV hybridization ( $>10$ cells/slide) as well as MGN's thus predicating the cause of the encephalopathy on CMV presence. Our findings of CMV hybridization in a variety of cell types (oligodendrocytes, neurons, astrocytes, ependymal cells, perivascular and endothelial cells) reflect similar findings in AIDS patients with encephalopathy. ${ }^{14}$ Our observation of the prominent presence of CMV infected endothelial and perivascular cells suggest that disruption of the blood brain barrier by viral infection may contribute to the encephalopathy. There is concomittent parenchymal CMV infection also which may also play a role in the encephalopathy.

CMV infection of the brain may have been underestimated in previous studies which employed immunohistochemical methods. ${ }^{15}$ We did not find evidence for CMV antigen in the same tissue studies that easily demonstrated viral genome by in situ hybridization. This suggests that in some cells the viral infection is latent or minimally productive.

We were impressed by the relatively high frequency of CMV gene localization in the NIC patients. Although the number of sections in which CMV appeared to be present was much less in the controls, CMV genomic material was found in at least $23 \%$ of these cases. The site of latency of CMV is uncertain. ${ }^{16}$ It can abortively infect macrophages and certain populations of lymphocytes. ${ }^{17}$ We propose that brain endothelial and perivascular cells might also serve as a reservoir for infection. Further studies will address this.

\section{ACKNOWLEDGEMENTS}

This work was supported by a Physicians' Services Incorporated grant.

\section{REFERENCES}

1. Maddrey WC, Van Thiel DH. Liver transplantation: an overview. Hepatology 1988; 8(4): 948-959.
2. Adams DH, Guson B, Honigsberger L, et al. Neurological complications following liver transplantation. Lancet 1987; 1:949-951.

3. Martinez AJ, Estol C, Faris A. Neurological complications of liver transplantation. Neurologic Clinics $1988 ; 6(2): 327-349$.

4. Kushne S, Dummer JS, Singh N, et al. Infections after liver transplantation. Medicine 1988; 67(2): 132-143.

5. Schneck SA. Neuropathological features of human organ transplantation. I: Probable cytomegalovirus infection. J Neuropathol Exp Neurol 1965; 24: 415-429.

6. Dorfman LJ. Cytomegalovirus encephalitis in adults. Neurology 1973; 23: 136-144.

7. Cordonnier C, Feuilhade F, Vernant JP, et al. Cytomegalovirus encephalitis occurring after bone marrow transplantation. Scand J Haematol 1983; 31: 248-252.

8. Wolber RA, Lloyd RV. Cytomegalovirus detection by nonisotope in situ hybridization and viral antigen immunostaining using a iwo-colour technique. Human Pathol 1988; 19(6): 736-741.

9. Weinshenker BG, Wilton S, Rice GPA. Phorbel ester-induced differentiation permits productive human cytomegalovirus infection in a monocytic cell line. J Immunol 1988; 140(5): 1625-1631.

10. Estol CJ, Lopez O, Brenner RP, et al. Seizures after liver transplantation: a clinicopathologic study. Neurol 1989; 39: 1297-1301

11. Petito CK, Cho ES, Leman BD, et al. Neuropathology of acquired immunodeficiency syndrome (AIDS): an autopsy review. J Neuropathol Exp Neurol 1986; 45: 635-646.

12. Anders K, Steinsapir KD, Iverson DJ, et al. Neuropathologic findings in the acquired immunodeficiency syndrome (AIDS). Clin Neuropathol 1986; 5: 1-20.

13. Navia BA, Cho ES, Petito CK, et al. The AIDS dementia complex: II neuropathology. Ann Neurol 1986; 19: 525-535.

14. Vinters HV, Kwok MK, Lo $\mathrm{HN}$ et al. Cytomegalovirus in the nervous system of patients with acquired immune deficiency syndrome. Brain 1989; 112(1): 246-268.

15. Wiley CA, Schrier RD, Denaro FJ, et al. Localization of cytomegalovirus proteins and genome during fulminant central nervous system infection in an AIDs patient. J Neuropathol Exp Neurol 1986; 45(2): 127-139.

16. Ho M, Cytomegalovirus-biology and infection. In: Greenbough WB, Merigan TC, eds. Current topics in infectious disease. New York: Plenum 1982; 174.

17. Rice GPA, Schrier RD, Oldstone MBA. Cytomegalovirus infects human lymphocytes and monocytes; virus expression is restricted to immediate-early gene products. Proc Natl Acad Sci USA 1984; 81:6134-6138. 